\title{
Tumor-derived biomimetic nanozyme with immune evasion ability for synergistically enhanced low dose radiotherapy
}

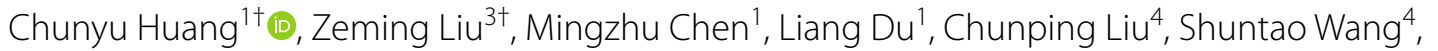
Yongfa Zheng ${ }^{2^{*}}$ and Wei Liu ${ }^{1^{*}}$

\begin{abstract}
High doses of radiation can cause serious side effects and efficient radiosensitizers are urgently needed. To overcome this problem, we developed a biomimetic nanozyme system (CF) by coating pyrite ( $\mathrm{FeS}_{2}$ ) into tumor-derived exosomes for enhanced low-dose radiotherapy (RT). CF system give $\mathrm{FeS}_{2}$ with immune escape and homologous targeting abilities. After administration, CF with both glutathione oxidase (GSH-OXD) and peroxidase (POD) activities can significantly lower the content of GSH in tumor tissues and catalyze intracellular hydrogen peroxide $\left(\mathrm{H}_{2} \mathrm{O}_{2}\right)$ to produce a large amount of. $\mathrm{OH}$ for intracellular redox homeostasis disruption and mitochondria destruction, thus reducing RT resistance. Experiments in vivo and in vitro showed that combining CF with RT (2 Gy) can provide a substantial suppression of tumor proliferation. This is the first attempt to use exosomes bionic $\mathrm{FeS}_{2}$ nanozyme for realizing low-dose RT, which broaden the prospects of nanozymes.
\end{abstract}

Keywords: Low-dose radiotherapy, Tumor-derived exosomes, Pyrite nanozyme, Mitochondrial damage, GSH depletion

\footnotetext{
*Correspondence: zyftgwz1986@163.com; wliu@whu.edu.cn

${ }^{\dagger}$ Chunyu Huang and Zeming Liu contributed equally to this work

${ }^{1}$ Key Laboratory of Artificial Micro- and Nano-Structures of Ministry

of Education, School of Physics and Technology, Wuhan University,

Wuhan 430072, People's Republic of China

2 Department of Oncology, Renmin Hospital of Wuhan University,

Wuhan 430060, Hubei, China

Full list of author information is available at the end of the article
}

(c) The Author(s) 2021. Open Access This article is licensed under a Creative Commons Attribution 4.0 International License, which permits use, sharing, adaptation, distribution and reproduction in any medium or format, as long as you give appropriate credit to the original author(s) and the source, provide a link to the Creative Commons licence, and indicate if changes were made. The images or other third party material in this article are included in the article's Creative Commons licence, unless indicated otherwise in a credit line to the material. If material is not included in the article's Creative Commons licence and your intended use is not permitted by statutory regulation or exceeds the permitted use, you will need to obtain permission directly from the copyright holder. To view a copy of this licence, visit http://creativecommons.org/licenses/by/4.0/. The Creative Commons Public Domain Dedication waiver (http://creativeco mmons.org/publicdomain/zero/1.0/) applies to the data made available in this article, unless otherwise stated in a credit line to the data. 


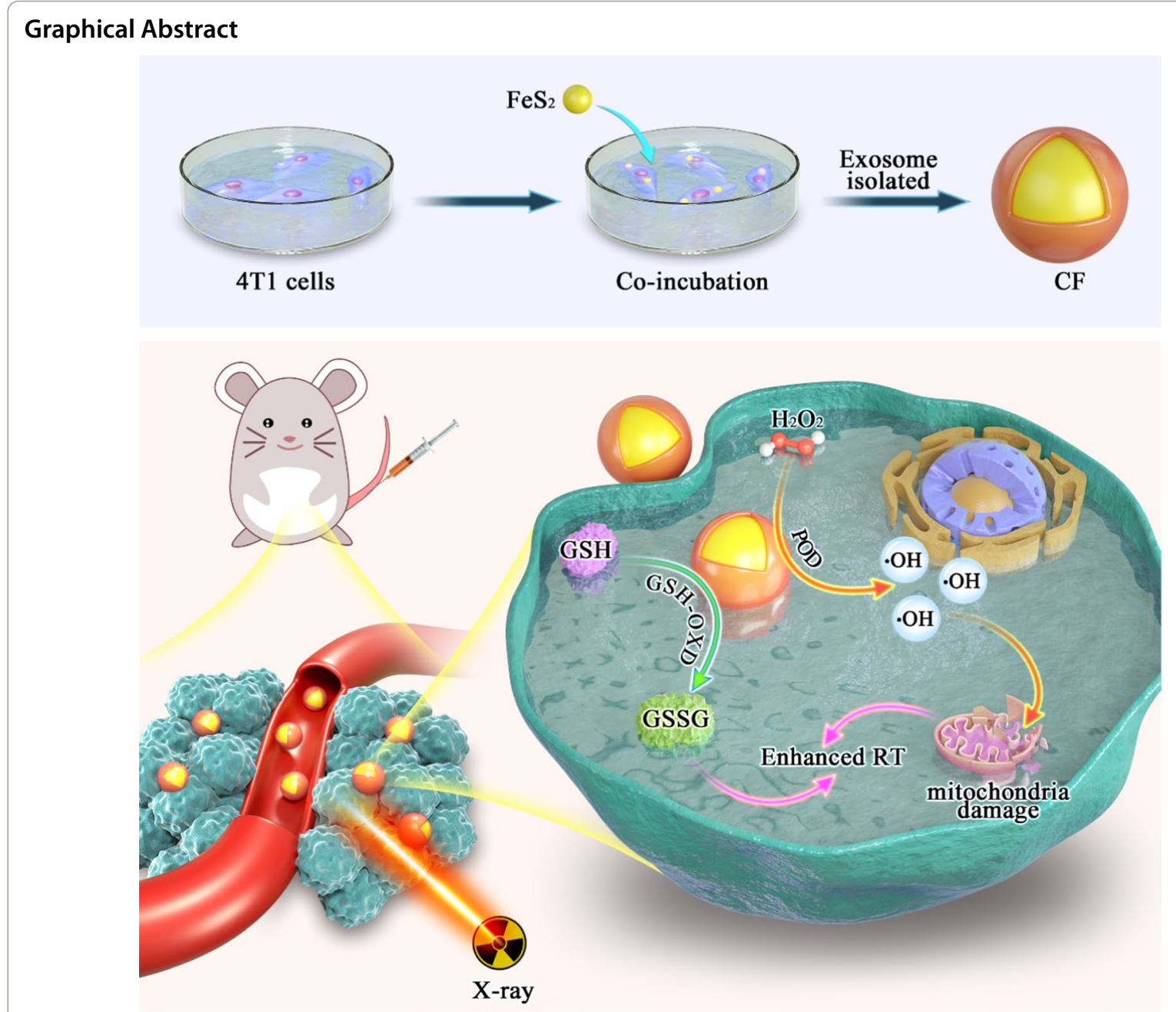

\section{Introduction}

Despite advances in scientific study, cancer, which can strike at any age, remains a severe threat to human life and health in today's society [1-3]. Radiotherapy (RT) is used to keep almost half of all cancer patients alive, either alone or in combination with other innovative treatments [4-6]. From a mechanical standpoint, RT relies on the use of high-energy X-rays or gamma rays to cause radiation-induced DNA damage or to stimulate the formation of large amounts of toxic reactive oxygen species (ROS), of which about $90 \%$ are produced by mitochondria [7]. Cell apoptosis occurs when the rate of ROS synthesis exceeds the cell's ability to neutralize these free radicals [8]. This allows tumor cells to be killed and the tumor to be abated. Long-term exposure to higher doses of radiation, on the other hand, can induce a variety of negative side effects, including fatigue, nausea and some digestive system diseases, etc. [9]. Furthermore, the tumor microenvironment (TME) in solid tumors exhibits high levels of GSH expression, which can inhibit antitumor RT $[10,11]$. Moreover, because GSH is a reducing agent, it can directly eliminate ROS which reduces the effectiveness of ROS-based therapies [12]. Therefore, reducing GSH level can effectively improve RT efficacy. GSH elimination can be accomplished through a variety of pathways due to the various pathways of glutathione metabolism and the various types of chemical reactions involving glutathione, and converting glutathione to an oxidation state through direct interactions has become one of the most commonly used methods for reducing glutathione levels [12-14]. Bao et al. for example, created a composite nanomaterial containing $\mathrm{MnO}_{2}$ to improve tumor hypoxia, alleviate and lower intracellular GSH levels, and therefore achieve radiation sensitization [15]. Qu et al. developed $\mathrm{MoS}_{2} @ \mathrm{AIBI}-\mathrm{PCM}$, a composite nanomaterial in which $\mathrm{MoS}_{2}$ can efficiently produce 


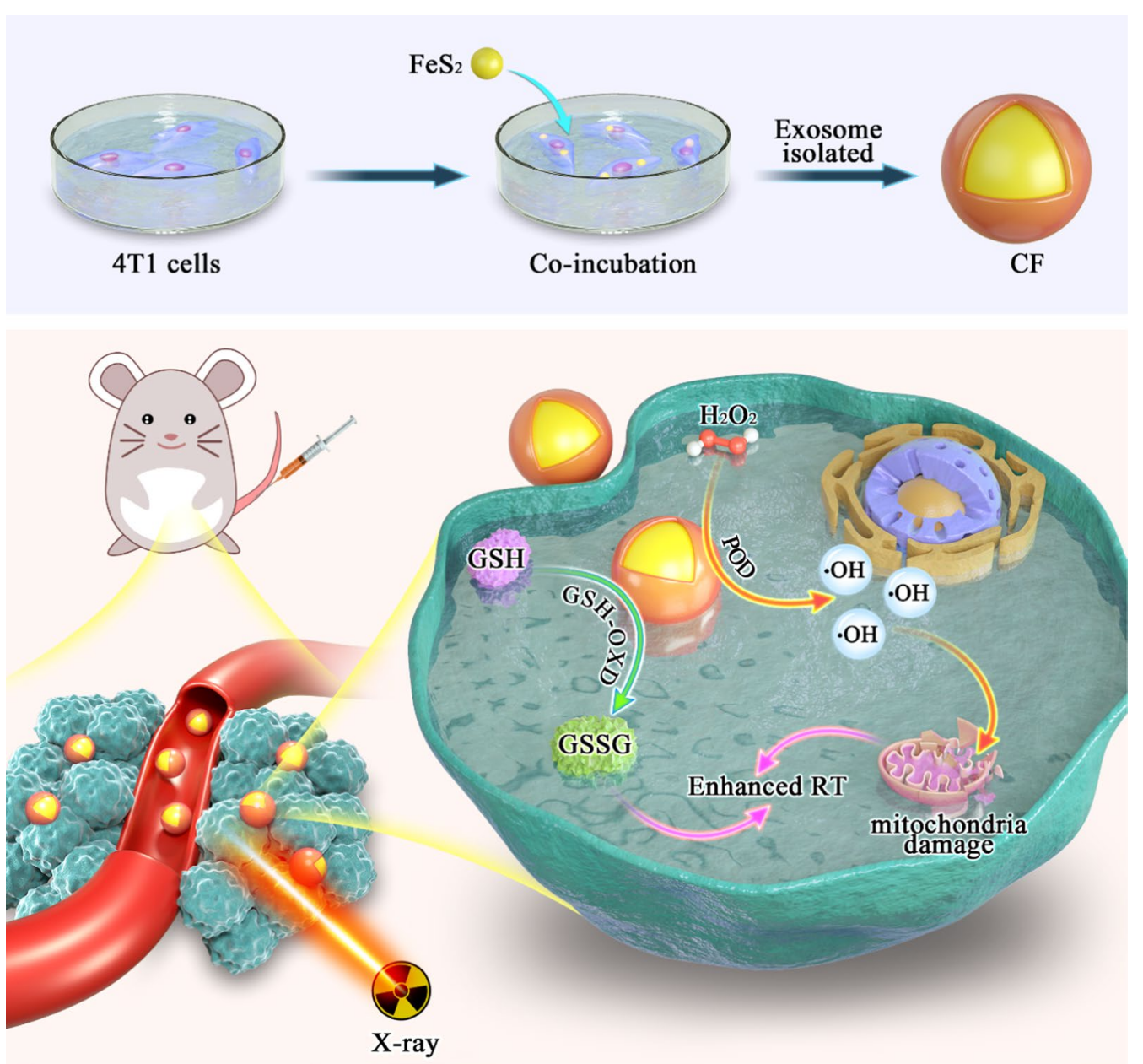

Scheme 1 Schematic illustration of tumor-derived biomimetic nanozyme with immune evasion ability for synergistically enhanced low dose radiotherapy

GSH oxidation without releasing hazardous metal ions, resulting in significant tumor death and good biocompatibility during therapy [16]. As a novel nano-materials, $\mathrm{FeS}_{2}$ nanozyme was verified to obtain both glutathione oxidase (GSH-OXD) and high-activity peroxidase (POD) [17]. Nanozymes have great advantages over natural enzymes [18-20]. $\mathrm{FeS}_{2}$ nanozyme can reduce GSH and catalyze $\mathrm{H}_{2} \mathrm{O}_{2}$ to produce sufficient $\cdot \mathrm{OH}$ to destroy mitochondria, which is expected to achieve a good synergistic effect combined with radiotherapy [21]. Despite the fact that these elements have proven effective against the GSH system, they are hardly to reach the tumor site [22]. These nanomaterials have a low immune escape capacity and are easily cleared by liver and kidney organs in the bloodstream; thus, their targeting ability and anti-tumor efficiency are considerably reduced [23, 24].

Cancer cell-derived exosomes (CDE) are endogenous vesicles ranging in size from 50 to $200 \mathrm{~nm}$ that are recovered from multivesicles or retrieved by incubating cell supernatants with appropriate separation kits [2527]. Because they may be generated from tumor cells and are less likely to elicit a clearance response than manufactured drug delivery platforms, such vesicles, given by cancer cells themselves, provide improved drug delivery prospects [28-30]. Furthermore, exosomes have a high level of non-immunogenicity, making them resistant to phagocytosis by macrophages [31]. Exosomes are also simpler to penetrate from blood arteries to tumor tissues for precise medication administration or nanomaterial delivery [32]. Therefore, it stimulates us to coat nanozymes with CDE to overcome tumor RT resistance.

The combined application of $\mathrm{FeS}_{2}$ nanozyme and CDE to augment low-dose radiation was originally reported in this work. By coating $\mathrm{FeS}_{2}$ with $\mathrm{CDE}$, a composite $\mathrm{CF}$ system was created (Scheme 1). Because CF has both homologous targeting and dual enzyme properties, the 

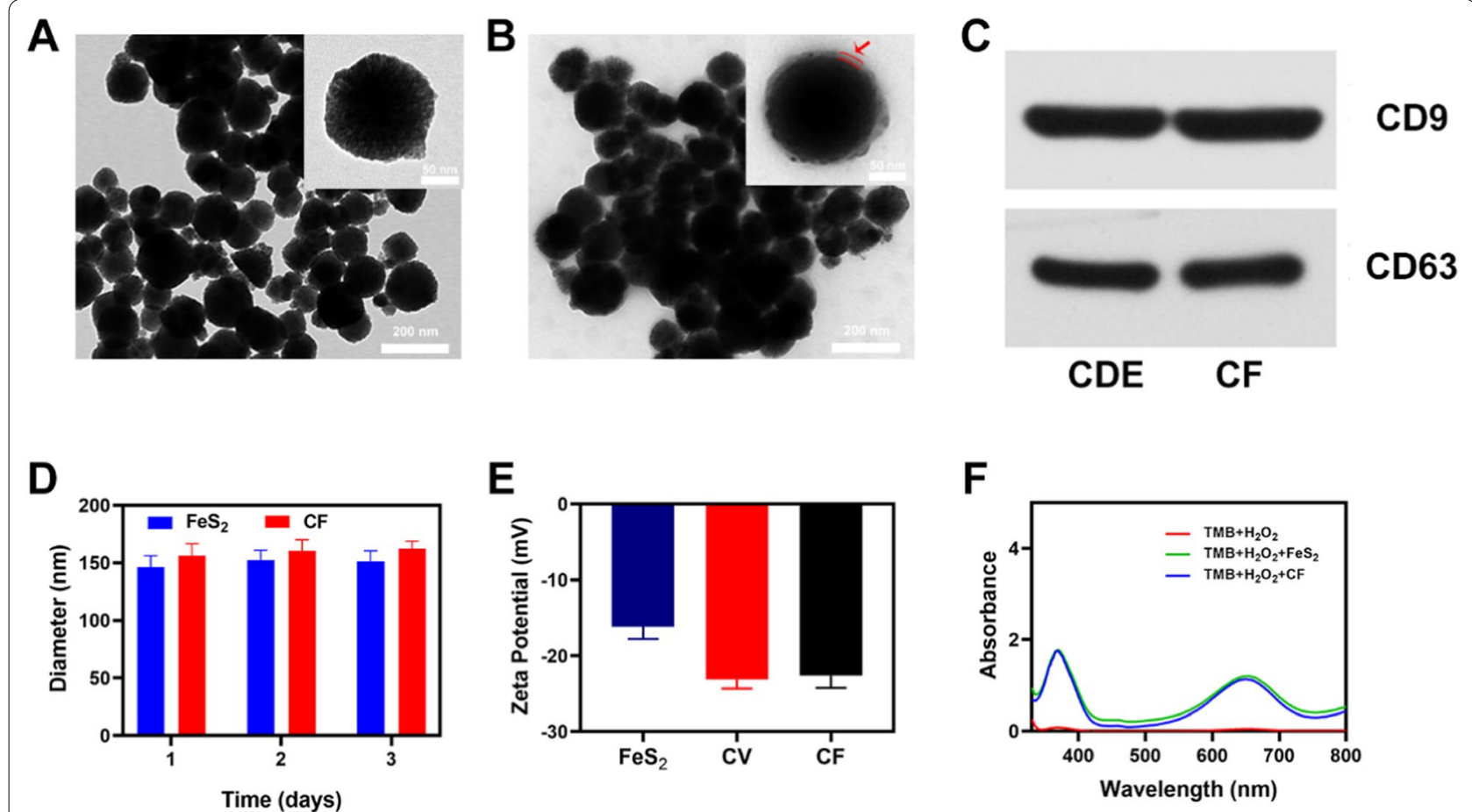

Fig. 1 TEM image of $\mathrm{FeS}_{2}(\mathbf{A})$ and $\mathrm{CF}$ (B) with the inset image showing the single image of corresponding materials. C Western blotting was used to measure the EXO markers CD9 and CD63. D Statistical graph of the measured diameter of $\mathrm{FeS}_{2}$ and CF. E Zeta potential values for FeS ${ }_{2}, \mathrm{CV}$, and CF nanovesicles. $\mathbf{F}$ UV - vis absorbance spectra and color changes of TMB in different reaction systems

exosome membrane can enhance $\mathrm{FeS}_{2}$ blood circulation time in vivo, and simultaneously help $\mathrm{FeS}_{2}$ actively targeting to tumor tissues. Subsequently, $\mathrm{FeS}_{2}$ nanozyme with GSH-OXD and POD activity can lower intracellular GSH levels. It catalyzes hydrogen peroxide in tumor cells to generate $\cdot \mathrm{OH}$, which disrupts redox equilibrium and kills mitochondria, resulting in radiation sensitization. It's important to mention that in the therapeutic process, we can get a powerful radiation sensitization with only $2 \mathrm{~Gy}$ of RT synergistic with CF, which is better than 6 Gy RT. CF system offers higher biological potential uses in the clinical. Finally, our findings broaden the use of exosomes and provide fresh insights into the development of exosom-based oncology therapeutic systems.

In this study, 4T1 cells were co-incubated with $\mathrm{FeS}_{2}$ materials, and exosome separation kits were used to obtain exocytosed CF, which was then employed to build a CF system. Transmission electron microscopy (TEM) was used to confirm the successful production of $\mathrm{CF}$ material. Compared with pure $\mathrm{FeS}_{2}$, CF was coated with a layer of exosome membrane with a small thickness (Fig. 1A, B), and this conclusion was further verified by western blotting assay (Fig. 1C). On the CDE membrane, the exosome protein markers $\mathrm{CD} 63$ and $\mathrm{CD} 9$ were identified $[21,28]$. On CF loaded with $\mathrm{FeS}_{2}$, the CD63 marker and another exosome marker (CD9) were also found, showing that the exosome membrane proteins on $\mathrm{CF}$ were not disturbed. Exosome membranes offer a lot of potential in the field of material delivery, and they're a lot better than standard drug delivery systems like erythrocyte membranes that don't have any targeting and don't improve the amount of material that gets into tumor tissues [33]. In addition, during the three days of pure $\mathrm{FeS}_{2}$ and $\mathrm{CF}$ stored in the phosphate buffer solution (PBS) environment $\left(4{ }^{\circ} \mathrm{C}\right)$, their particle sizes almost did not change significantly. During the 3 days, the particle sizes of $\mathrm{FeS}_{2}$ were $146.2 \pm 1.6 \mathrm{~nm}, 152.6 \pm 1.8 \mathrm{~nm}$, and $151.0 \pm 1.4 \mathrm{~nm}$, respectively, while the particle sizes of CF were $153 \pm 2.1 \mathrm{~nm}, 159.4 \pm 2.5 \mathrm{~nm}$, and $157.5 \pm 1.9 \mathrm{~nm}$ (Fig. 1D), which also indicates that both $\mathrm{FeS}_{2}$ and hybrid CF have good stability and can be used in subsequent biological experiments. This quality is unquestionably critical, as many materials have good properties, but their instability will prevent them from being used [34]. The Zeta potential of different particles was shown in Fig. 1E, with cancer cell vesicle (CV) and CF having zeta potentials of roughly about -23.1 and -22.6 , respectively. We investigated POD-like activity of $\mathrm{FeS}_{2}$ and CF based on the oxidation of $3,3^{\prime}, 5,5^{\prime}$-tetramethylbenzidine (TMB) in the presence of $\mathrm{H}_{2} \mathrm{O}_{2}$. The results show that both pure $\mathrm{FeS}_{2}$ and $\mathrm{FeS}_{2}$ encapsulated by exosome membrane can catalyze $\mathrm{H}_{2} \mathrm{O}_{2}$ to produce $\cdot \mathrm{OH}$ (Fig. $1 \mathrm{~F}$ ). These findings 


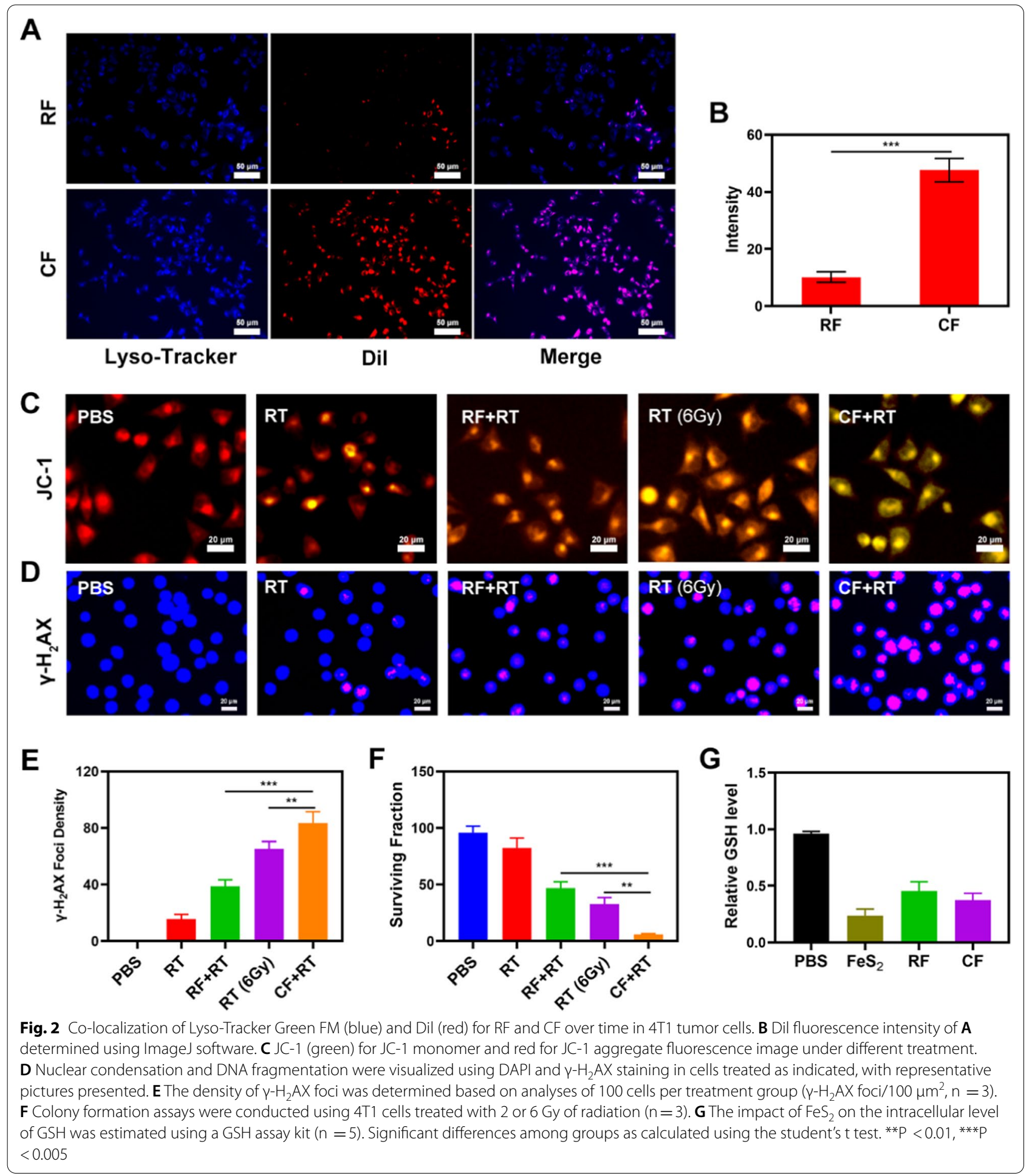

show that CF has an exosomal membrane structure and that it will be endocytosed into $4 \mathrm{~T} 1$ tumor cells via extracellular action to alter the tumor microenvironment.

The CF system is well-structured and characterized in terms of performance. In vitro anti-tumor trials are currently underway. Although $\mathrm{FeS}_{2}$ can alter the ecological balance of the cancer cells and so enhance the efficacy of radiation, it can only do so when it is present in the tumor tissue. The immune system may recognize diverse foreign invaders' stimuli, and a portion of that 


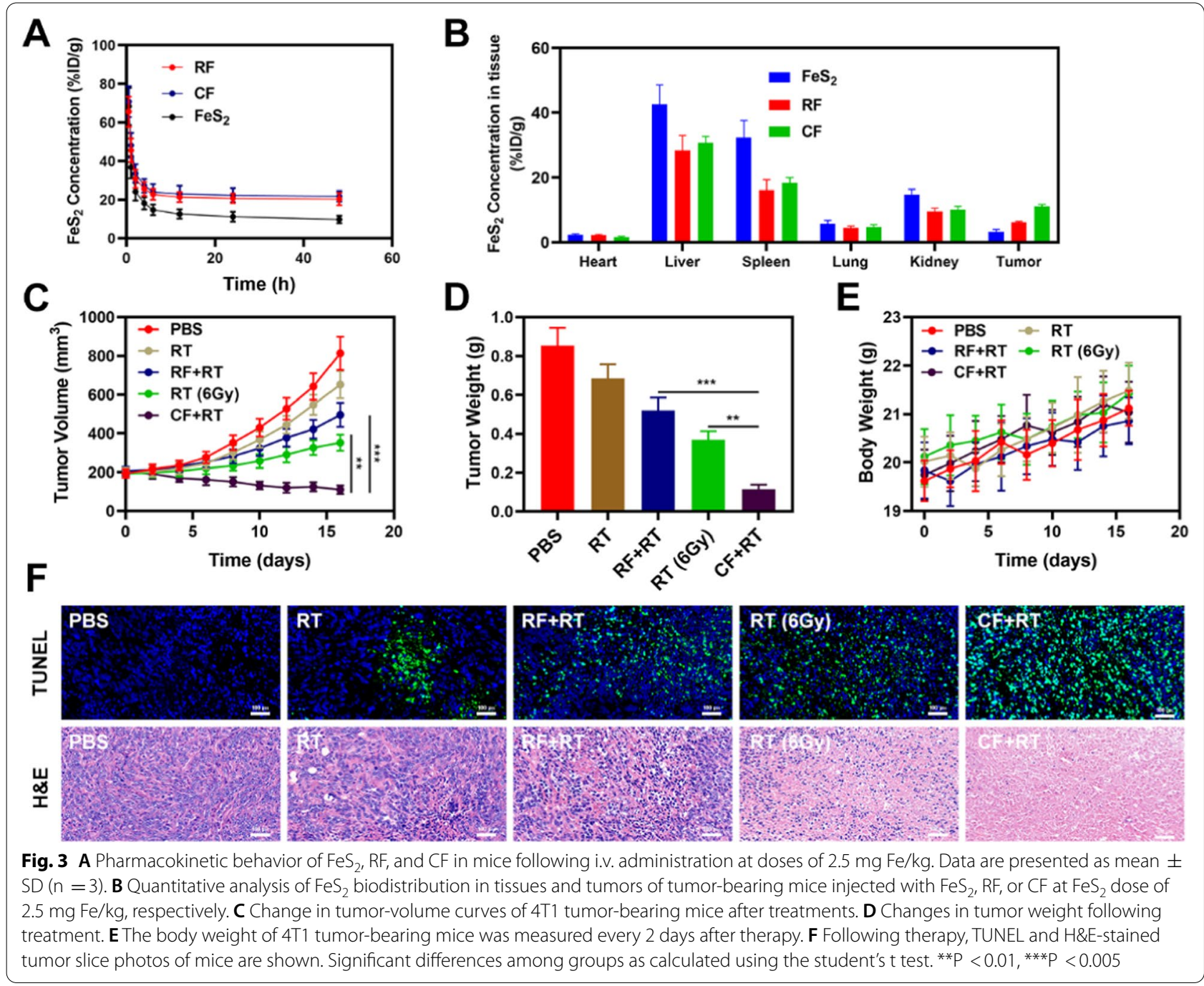

stimulation can activate the immune response, resulting in immunity, while preventing other stimuli, resulting in tolerance [35]. Due to homology, which is connected to exosome membrane proteins, the immune system does not attack the exosomes released by cancer cells. Nanomaterial-coated exosomes can be directed to tumor cells, recognized by cancer cells, and engorged to release nano-material, whereas other drug delivery systems that do not have targeting capabilities. This internalization effect can be tracked by staining $\mathrm{CF}$ and red blood cell membranes coated $\mathrm{FeS}_{2}$ (RF) nanovesicles with Dil dye and co-incubation with 4T1 cells and staining with commercial lysosome Lyso-Tracker Green probe to verify the ability of CF to be internalized by tumor cells in vitro (colocalization assay). For comparison, red cell membranes were coated with $\mathrm{FeS}_{2}$ to form RF. After $2 \mathrm{~h}$ incubation, it was clear that a significant quantity of $\mathrm{CF}$ had been endocytosed, whereas RF only had a partial Dil fluorescent impact (Fig. 2A, B), demonstrating that exosomes might be employed as a perfect carrier of $\mathrm{FeS}_{2}$ to target tumor tissues. As shown in Additional file 1: Figure S2, hemolysis assay verified that $\mathrm{CF}$ is stable in blood, indicating good biocompatibility of CF. In order to demonstrated the universality of exosome systems, we then prepared manganese dioxide nanozyme $\left(\mathrm{MnO}_{2}\right)$, as shown in Additional file 1: Figure S3, and used CDE to coat $\mathrm{MnO}_{2}$ (CM) (Additional file 1: Figure S4). Furthermore, during the several days of $\mathrm{MnO}_{2}$ and MF stored in the PBS environment $\left(4{ }^{\circ} \mathrm{C}\right)$, the diameter of $\mathrm{MnO}_{2}$ and $\mathrm{CM}$ has barely changed (Additional file 1: Figure S5). $\mathrm{MnO}_{2}$ has good catalase activity (CAT), which can catalyze $\mathrm{H}_{2} \mathrm{O}_{2}$ into $\mathrm{O}_{2}$. CM also has good CAT activity, indicating that exosome membrane does not affect the enzyme activity (Additional file 1: Figure S6). Even at high concentrations, the number of $\mathrm{MnO}_{2}$ swallowed by $4 \mathrm{~T} 1$ cells was low (Additional file 1: Figure S8). As $\mathrm{MnO}_{2}$ can achieve 
radiotherapy sensitization by alleviating tumor hypoxic microenvironment, while CM can actively target cells, as shown in Additional file 1: Figure S7, the colony formation assays also proves that $\mathrm{CM}$ has better synergetic cell killing effect compared with $\mathrm{MnO}_{2}$. Then, the changes of mitochondrial membrane potential (MMP) in tumor cells were monitored by JC-1 (5,5',6,6'-tetrachloro-1,1',3,3'tetraethyl-imidacarbocyanine) probe method. The JC dye normally builds up in the mitochondria, where it clumps together to give a red fluorescence. When mitochondria are injured and MMP levels are low, however, the JC monomer is released into the cytoplasm, resulting in green fluorescence [36]. Figure $2 \mathrm{C}$ demonstrates that cells treated with $\mathrm{FeS}_{2}+\mathrm{RT}$ had a high green/red fluorescence ratio, which is consistent with the reduced mitochondrial damage generated by $\mathrm{FeS}_{2}$. $\mathrm{FeS}_{2}$ will enter TME with twofold enzyme activity once CF is endocytosed by tumor cells, reducing GSH content while also catalyzing enough $\mathrm{H}_{2} \mathrm{O}_{2}$ to generate $\cdot \mathrm{OH}$ in situ, causing significant mitochondrial damage. Radiotherapy ought to be more sensitive as a result of mitochondrial injury. Doublestranded DNA breaks (DSB) in tumor cells when exposed to radiation, which provides insights into the radiation sensitization, and measuring the fluorescence intensity of $\gamma-\mathrm{H} 2 \mathrm{AX}$, is a good and intelligent way to verify the DSB formation after cell damage [37]. Therefore, we detected $\mathrm{H} 2 \mathrm{AX}$ foci in the nucleus after treatment in different groups. After 2 Gy of radiotherapy, there was substantial DNA damage, and the DSB effect increased as the dose was increased. It's important to mention that 2 Gy radiation mixed with RF only got about $40 \% \gamma-\mathrm{H} 2 \mathrm{AX}$ formation, but 2 Gy paired with CF got up to $76.8 \% \gamma$-H2AX foci development. The synergistic effect of the targeting ability of the exosome membrane, the dual nano enzyme activity of $\mathrm{FeS}_{2}$, and radiation sensitization was linked to the uniform and substantial difference between each experimental group. Furthermore, Colony formation assays revealed that the control group's cell viability was largely unaltered, whereas the RT with RF group had moderate tumor growth inhibition (Fig. 2F). CF + RT system had the best tumor growth inhibition rate (90\%), there are significant differences compared with each other experiment group, indicating that $\mathrm{CF}$ mediated improved $\cdot \mathrm{OH}$ content of TME can effectively exert influence on mitochondria and thus enhance the RT effect to realize tumor growth inhibition. Figure $2 \mathrm{G}$ also verifies that our $\mathrm{FeS}_{2}$ has a good effect on GSH consumption. Together, these results drive our continued exploration of anti-tumor efficacy in vivo.

Given that in vitro testing of the tumor-killing action revealed significant potential. We investigate how $\mathrm{CF}$ effects can be amplified in vivo. As a result, we conducted in vivo pharmacokinetic studies to see how exosome membranes affect blood retention. Mice were given $\mathrm{FeS}_{2}, \mathrm{RF}$, or $\mathrm{CF}$ at a dose of $2.5 \mathrm{mg} \mathrm{Fe} / \mathrm{kg}$ intravenously (Fig. 3A). Both RF and CF demonstrated a greater effect

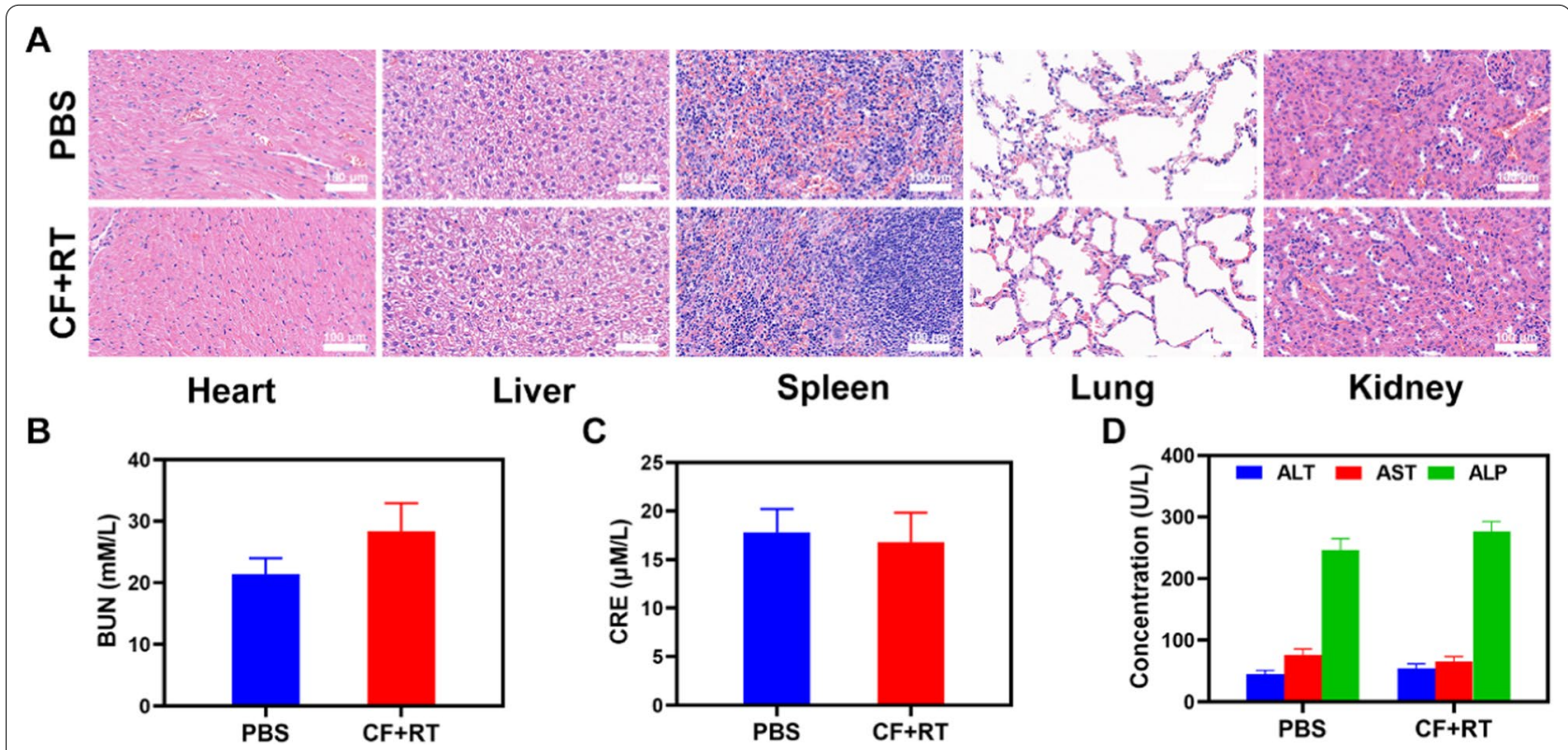

Fig. 4 Result of in vivo safety experiments. A Histopathological analysis results (H\&E) stained images of the major organs, heart, lung, liver, kidneys, and spleen, of mice that were exposed to different treatments 16 days post-injection under laser irradiation. Blood biochemistry data including kidney function markers: $\mathbf{B}$ liver function markers: BUN, C CRE, and D ALT, ALP, and AST after various treatments 
on blood retention when compared to the pure $\mathrm{FeS}_{2}$ group. Although the erythrocyte membrane coating protected from the attack of the immune system, it lacked tumor targeting, and the accumulation of $\mathrm{FeS}_{2}$ in tumor tissues was only slightly increased, with the CF group having the most visible tumor accumulation. Following that, we investigated the biological dispersion (Fig. 3B). After $12 \mathrm{~h}$ of administration, the $\mathrm{FeS}_{2}$ mainly accumulated in the liver and spleen of the $\mathrm{FeS}_{2}$ group of mice, whereas the CF group demonstrated good tumor targeting and little organ accumulation, demonstrating the exosome membrane's targeting capabilities. In order to verify the targeting ability of drugs-loaded exosomes, we conducted in vivo pharmacokinetic studies using DOX loaded erythrocyte exosomes (RBC-EXO@DOX) and CDE (CDE-EXO@DOX), respectively. Simultaneously, DOX loaded CF was prepared (CF@DOX). As shown in Additional file 1: Figures S9, S10, RBC-EXO@DOX and CDE both have long circulation. However, RBC-EXO@ DOX accumulates less in tumor tissues compared with CDE-EXO@DOX and CF@DOX (Additional file 1: Figure S10). This result shows that tumor-derived exosomes have good tumor targeting. Following that, we looked at the efficacy of CF-mediated anti-tumor therapy in mice with $4 \mathrm{~T} 1$ tumors. BALB/c mice were subcutaneously injected with $1 \times 10^{6} 4 \mathrm{~T} 1$ cells into the right flank to determine the primary effect of the CF. When the primary tumor volume reached $200 \mathrm{~mm}^{3}$, the mice were divided into groups and treated. Tumor-bearing mice were divided randomly into 5 groups (each group included 5 mice): (1) control (PBS); (2) radiotherapy (RT, 2 Gy); (3) RF + RT; (4) high dose RT (6 Gy); (5) $\mathrm{CF}+\mathrm{RT}$. The $\mathrm{FeS}_{2}$ concentration was $5 \mathrm{mg} / \mathrm{kg}$ in groups 3 , and 5. For 16 days, the treatment was given every 4 days. The tumor volumes of the control and RT treated groups increased rapidly during the 2 weeks of treatment, as shown in Fig. 3C. The RF + RT group likewise had a nearly moderate tumor-suppressive effect. When these nanomaterials are injected into the caudal vein, when the RF circulates to the tumor tissue and is endocytosed by tumor cells, $\mathrm{FeS}_{2}$ is released to the TME, playing the equivalent therapeutic impact. The CF + RT system, which included both $\mathrm{FeS}_{2}$, had the most potent therapeutic impact, with tumor volume growth curves nearly totally suppressed during therapy. The tumor mass in mice was also consistent with the volume curve (Fig. 3D). No weight changes were detected in the treatment group during this study, indicating that the treatment did not cause any significant systemic toxicity (Fig. 3E), which is significant because many treatments are associated with extremely systemic toxicity, which is extremely detrimental to the future clinical application of the material. We collected tumor tissue slices for staining. TUNEL and
H\&E staining (Fig. 3F) indicated the presence of a high level of cell necrosis in the CF combined RT therapy group. Furthermore, as shown in Fig. 4, after the treatment of mice's vital organs (heart, liver, spleen, lungs, and kidney) without any inflammation and damage in the body, liver, and kidney indices were also normal. Many nanomaterials have high therapeutic efficacy, however, they are also associated with systemic toxicity, limiting their future clinical applications. The in vivo results show that our innovative combined therapy not only achieves an excellent therapy with biological safety but also enhances tumor $\cdot \mathrm{OH}$ content and reinforces the effect of RT with substantial CF-enhanced therapy.

\section{Conclusion}

Finally, we developed a unique oxidative stress destroyer to enable better radiotherapy. The CF can decrease the content of GSH in the tumor microenvironment and catalyze $\mathrm{H}_{2} \mathrm{O}_{2}$ to produce a large amount of $\cdot \mathrm{OH}$, the exosome membrane can render $\mathrm{FeS}_{2}$ with admirable homologous targeting ability, and all of these elements have an enhanced ability to low-dose RT. Both the in vitro and in vivo studies suggest that our method has a good tumor inhibition effect. Importantly, during treatment, our prepared system showed no evident side effects. In the future, we will continue to investigate the biological applications of the combination of $\mathrm{FeS}_{2}$ and other unique vehicles, as well as refine our treatment strategy by incorporating additional nanotechnology and new technologies.

\section{Supplementary Information}

The online version contains supplementary material available at https://doi. org/10.1186/s12951-021-01182-y.

\section{Additional file 1: Figure S1. Nanoparticle uptake by RAW 264.7 cells at different incubated concentration ( $\mathrm{FeS}_{2}$ dose of 25,50 and $100 \mu \mathrm{g} / \mathrm{mL}$ ). Data are presented as mean $\pm \mathrm{SD}(\mathrm{n}=3)$. Figure $\mathbf{S 2}$. Hemolysis ratio of CF at different $\mathrm{FeS}_{2}$ concentrations. Figure S3.TEM image of $\mathrm{MnO}_{2}$. Figure S4.TEM image of CM. Figure S5. Statistical graph of the measured diameter of $\mathrm{MnO}_{2}$ and $\mathrm{CM}$. Data are presented as mean $\pm \mathrm{SD}(\mathrm{n}=3)$. Figure S6. Oxygen generation under different conditions measured using a dissolved oxygen meter. Figure S7. Nanoparticles uptake by 4T1 cells at different concentration. Data are presented as mean $\pm \operatorname{SD}(n=3)$. Figure S8. Colony formation assays were conducted using 4T1 cells with different treatment $(n=3)$. Figure S9. Pharmacokinetic behavior of RBC-EXO@ DOX,CDE-EXO@DOX, and CF@DOX in mice following i.v. administra- tion. Data are presented as mean \pm SD $(n=3)$. Figure S10. Quantitative analysis of DOX biodistribution in tissues and tumors of tumor-bearing mice injected with different formulations. Data are presented as mean \pm $\mathrm{SD}(\mathrm{n}=3)$.}

\section{Acknowledgements}

This work was supported by the National Natural Science Foundation of China (Grant No. 81703035, No. 31971166). This work was supported by Key Projects of Science and Technology to Help the Economy 2020 (Grant No. SQ2020YFF0426493), the Basic Research Projects of Shenzhen Knowledge Innovation Program (JCYJ20180302173424902), and the Frontier Projects of 
Applied Foundation in Wuhan (2019010701011386). The animal experiments were carried out according to the protocol approved by the Ministry of Health in the People's Republic of PR China and were approved by the Administrative Committee on Animal Research of the Wuhan University.

\section{Authors' contributions}

HC and CM: conceptualization; HC and DL: writing review and editing; LW supervision and resources. LZ, LC and W S visualization. LW and ZY funding acquisition. All authors have read and approved the final manuscript.

\section{Availability of data and materials}

All the original data are available upon reasonable request for correspondence authors.

\section{Declarations}

\section{Competing interests}

The authors declare no competing interests.

\begin{abstract}
Author details
'Key Laboratory of Artificial Micro- and Nano-Structures of Ministry of Education, School of Physics and Technology, Wuhan University, Wuhan 430072, People's Republic of China. ${ }^{2}$ Department of Oncology, Renmin Hospital of Wuhan University, Wuhan 430060, Hubei, China. ${ }^{3}$ Department of Plastic and Cosmetic Surgery, Tongji Hospital, Tongji Medical College, Huazhong University of Science and Technology, Wuhan 430030, China. ${ }^{4}$ Department of Breast and Thyroid Surgery, Union Hospital, Tongji Medical College, Huazhong University of Science and Technology, Wuhan 430022, China.
\end{abstract}

Received: 23 September 2021 Accepted: 4 December 2021

Published online: 28 December 2021

\section{References}

1. Zhu D, Lyu M, Jiang W, Suo M, Huang Q, Li K. A biomimetic nanozyme/ camptothecin hybrid system for synergistically enhanced radiotherapy. J Mater Chem B. 2020:8(24):5312-9.

2. Gao F, Tang Y, Liu WL, Zou MZ, Huang C, Liu CJ, Zhang XZ. Intra/extracellular lactic acid exhaustion for synergistic metabolic therapy and immunotherapy of tumors. Adv Mater. 2019;31(51):1904639.

3. Liu LH, Zhang YH, Qiu WX, Zhang L, Gao F, Li B, Xu L, Fan JX, Li ZH, Zhang XZ. Dual-stage light amplified photodynamic therapy against hypoxic tumor based on an $\mathrm{O}_{2}$ self-sufficient nanoplatform. Small. 2017;13(37):1701621.

4. Song G, Cheng L, Chao Y, Yang K, Liu Z. Emerging nanotechnology and advanced materials for cancer radiation therapy. Adv Mater. 2017;29(32):1700996.

5. Liu T, Yang K, Liu Z. Recent advances in functional nanomaterials for X-ray triggered cancer therapy. Prog Nat Sci. 2020;30(5):567-76.

6. Xie J, Gong L, Zhu S, Yong Y, Gu Z, Zhao Y. Emerging strategies of nanomaterial-mediated tumor radiosensitization. Adv Mater. 2019;31(3):e1802244

7. Li N, Yu L, Wang J, Gao X, Chen Y, Pan W, Tang B. A mitochondria-targeted nanoradiosensitizer activating reactive oxygen species burst for enhanced radiation therapy. Chem Sci. 2018;9(12):3159-64.

8. Noh J, Kwon B, Han E, Park M, Yang W, Cho W, Yoo W, Khang G, Lee D. Amplification of oxidative stress by a dual stimuli-responsive hybrid drug enhances cancer cell death. Nat Commun. 2015;6:6907.

9. Rocca JD, Werner ME, Kramer SA, Huxford-Phillips RC, Sukumar R, Cummings ND, Vivero-Escoto JL, Wang AZ, Lin W. Polysilsesquioxane nanoparticles for triggered release of cisplatin and effective cancer chemoradiotherapy. Nanomedicine. 2015;11(1):31-8.

10. Yin W, Li J, Ke W, Zha Z, Ge Z. Integrated nanoparticles to synergistically elevate tumor oxidative stress and suppress antioxidative capability for amplified oxidation therapy. ACS Appl Mater Interfaces. 2017;9(35):29538-46.

11. Cheng X, Xu HD, Ran HH, Liang G, Wu FG. Glutathione-depleting nanomedicines for synergistic cancer therapy. ACS Nano. 2021;15(5):8039-68.
12. Lan J-S, Liu L, Zeng R-F, Qin Y-H, Hou J-W, Xie S-S, Yue S, Yang J, Ho RJY, Ding Y, Zhang T. Tumor-specific carrier-free nanodrugs with GSH depletion and enhanced ROS generation for endogenous synergistic anti-tumor by a chemotherapy-photodynamic therapy. Chem Eng J. 2021:407(1):127212

13. Liu Y, Zhai S, Jiang X, Liu Y, Wang K, Wang C, Zhang M, Liu X, Bu W. Intracellular mutual promotion of redox homeostasis regulation and iron metabolism disruption for enduring chemodynamic therapy. Adv Funct Mater. 2021:31(17):2010390.

14. Wu Y, Guo T, Qiu Y, Lin Y, Yao Y, Lian W, Lin L, Song J, Yang H. An inorganic prodrug, tellurium nanowires with enhanced ROS generation and GSH depletion for selective cancer therapy. Chem Sci. 2019;10(29):7068-75.

15. Lyu M, Zhu D, Kong X, Yang Y, Ding S, Zhou Y, Quan H, Duo Y, Bao Z. Glutathione-depleting nanoenzyme and glucose oxidase combination for hypoxia modulation and radiotherapy enhancement. Adv Healthc Mater. 2020;9(11):e1901819.

16. Wu S, Liu X, Ren J, Qu X. Glutathione depletion in a benign manner by MoS2-based nanoflowers for enhanced hypoxia-irrelevant free-radicalbased cancer therapy. Small. 2019;15(51):e1904870.

17. Meng X, Li D, Chen L, He H, Wang Q, Hong C, He J, Gao X, Yang Y, Jiang B, Nie G, Yan X, Gao L, Fan K. High-performance self-cascade pyrite nanozymes for apoptosis-ferroptosis synergistic tumor therapy. ACS Nano. 2021;15(3):5735-51.

18. Zhu D, Zheng Z, Luo G, Suo M, Li X, Duo Y, Tang BZ. Single injection and multiple treatments: an injectable nanozyme hydrogel as AIEgen reservoir and release controller for efficient tumor therapy. Nano Today. 2021:37:101091.

19. Zhang R, Yan X, Fan K. Nanozymes inspired by natural enzymes. Acc Mater Res. 2021;2(7):534-47.

20. Tang G, He J, Liu J, Yan X, Fan K. Nanozyme for tumor therapy: surface modification matters. Exploration. 2021;1(1):75-89.

21. Zhu D, Liu Z, LiY, Huang Q, Xia L, Li K. Delivery of manganese carbonyl to the tumor microenvironment using tumor-derived exosomes for cancer gas therapy and low dose radiotherapy. Biomaterials. 2021;274:120894.

22. Zhu Y, Shi H, LiT, Yu J, Guo Z, Cheng J, Liu Y. A dual functional nanoreactor for synergistic starvation and photodynamic therapy. ACS Appl Mater Interfaces. 2020;12(16):18309-18.

23. Rao L, Meng QF, Bu LL, Cai B, Huang Q, Sun ZJ, Zhang WF, Li A, Guo SS, Liu W, Wang TH, Zhao XZ. Erythrocyte membrane-coated upconversion nanoparticles with minimal protein adsorption for enhanced tumor imaging. ACS Appl Mater Interfaces. 2017;9(3):2159-68.

24. Su J, Sun H, Meng Q, Zhang P, Yin Q, Li Y. Enhanced blood suspensibility and laser-activated tumor-specific drug release of theranostic mesoporous silica nanoparticles by functionalizing with erythrocyte membranes. Theranostics. 2017;7(3):523-37.

25. Wang D, Yao Y, He J, Zhong X, Li B, Rao S, Yu H, He S, Feng X, Xu T, Yang $B$, Yong T, Gan L, Hu J, Yang X. Engineered cell-derived microparticles Bi2Se3/DOX@MPs for imaging guided synergistic photothermal/lowdose chemotherapy of cancer. Adv Sci. 2020;7(3):1901293.

26. Yang Z, Shi J, Xie J, Wang Y, Sun J, Liu T, Zhao Y, Zhao X, Wang X, Ma Y, Malkoc V, Chiang C, Deng W, Chen Y, Fu Y, Kwak KJ, Fan Y, Kang C, Yin C, Rhee J, Bertani P, Otero J, Lu W, Yun K, Lee AS, Jiang W, Teng L, Kim BYS, Lee LJ. Large-scale generation of functional mRNA-encapsulating exosomes via cellular nanoporation. Nat Biomed Eng. 2020;4(1):69-83.

27. Jia G, Han Y, An Y, Ding Y, He C, Wang X, Tang Q. NRP-1 targeted and cargo-loaded exosomes facilitate simultaneous imaging and therapy of glioma in vitro and in vivo. Biomaterials. 2018;178:302-16.

28. Guo W, Li Y, Pang W, Shen H. Exosomes: a potential therapeutic tool targeting communications between tumor cells and macrophages. Mol Ther. 2020;28(9):1953-64.

29. Zhu D, Duo Y, Meng S, Zhao Y, Xia L, Zheng Z, Li Y, Tang BZ. Tumorexocytosed exosome/aggregation-induced emission luminogen hybrid nanovesicles facilitate efficient tumor penetration and photodynamic therapy. Angew Chem. 2020;59:2-10.

30. Zhu D, Lyu M, Huang Q, Suo M, Liu Y, Jiang W, Duo Y, Fan K. Stellate plasmonic exosomes for penetrative targeting tumor NIR-II thermoradiotherapy. ACS Appl Mater Interfaces. 2020;12(33):36928-37.

31. Zhu DM, Duo YH, Suo M, Zhao YH, Xia LG, Zheng Z, Li Y, Tang BZ. Tumorexocytosed exosome/aggregation-induced emission luminogen hybrid nanovesicles facilitate efficient tumor penetration and photodynamic therapy. Angew Chem-Int Edit. 2020;59(33):13836-43. 
32. Yong T, Zhang X, Bie N, Zhang H, Zhang X, Li F, Hakeem A, Hu J, Gan L, Santos HA, Yang $X$. Tumor exosome-based nanoparticles are efficient drug carriers for chemotherapy. Nat Commun. 2019;10(1):3838.

33. Taylor DD, Gercel-Taylor C. MicroRNA signatures of tumor-derived exosomes as diagnostic biomarkers of ovarian cancer. Gynecol Oncol. 2008;110(1):13-21.

34. Ouyang J, Deng L, Chen W, Sheng J, Liu Z, Wang L, Liu YN. Two dimensional semiconductors for ultrasound-mediated cancer therapy: the case of black phosphorus nanosheets. Chem Commun. 2018:54(23):2874-7.

35. Liu Y, Zhen W, Wang Y, Song $\mathrm{S}$, Zhang $\mathrm{H} . \mathrm{Na}_{2} \mathrm{~S}_{2} \mathrm{O}_{8}$ nanoparticles trigger antitumor immunotherapy through reactive oxygen species storm and surge of tumor osmolarity. J Am Chem Soc. 2020;142(52):21751-7.

36. Fu S, Yang R, Zhang L, Liu W, Du G, Cao Y, Xu Z, Cui H, Kang Y, Xue P. Biomimetic CoO@AuPt nanozyme responsive to multiple tumor microenvironmental clues for augmenting chemodynamic therapy. Biomaterials. 2020;257:120279.

37. Begg AC, Stewart FA, Vens C. Strategies to improve radiotherapy with targeted drugs. Nat Rev Cancer. 2011;11(4):239-53.

\section{Publisher's Note}

Springer Nature remains neutral with regard to jurisdictional claims in published maps and institutional affiliations.

- fast, convenient online submission

- thorough peer review by experienced researchers in your field

- rapid publication on acceptance

- support for research data, including large and complex data types

- gold Open Access which fosters wider collaboration and increased citations

- maximum visibility for your research: over $100 \mathrm{M}$ website views per year

At BMC, research is always in progress.

Learn more biomedcentral.com/submissions 